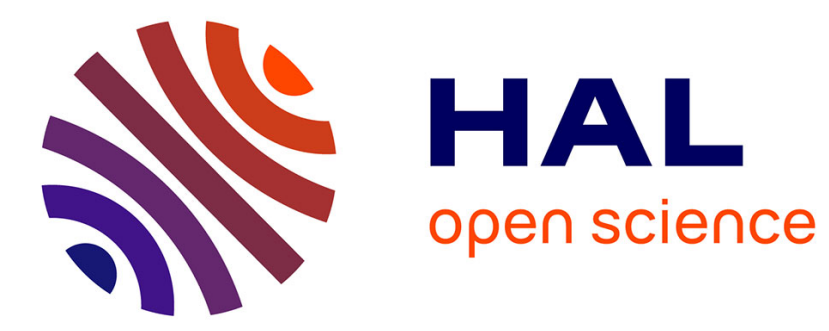

\title{
Equilibrium mechanics of monolayered epithelium
}

Jure Derganc, S. Saša Svetina, B. Boštjan Žekš

\section{To cite this version:}

Jure Derganc, S. Saša Svetina, B. Boštjan Žekš. Equilibrium mechanics of monolayered epithelium. Journal of Theoretical Biology, 2009, 260 (3), pp.333. 10.1016/j.jtbi.2009.06.021 . hal-00554631

\section{HAL Id: hal-00554631 \\ https://hal.science/hal-00554631}

Submitted on 11 Jan 2011

HAL is a multi-disciplinary open access archive for the deposit and dissemination of scientific research documents, whether they are published or not. The documents may come from teaching and research institutions in France or abroad, or from public or private research centers.
L'archive ouverte pluridisciplinaire HAL, est destinée au dépôt et à la diffusion de documents scientifiques de niveau recherche, publiés ou non, émanant des établissements d'enseignement et de recherche français ou étrangers, des laboratoires publics ou privés. 


\section{Author's Accepted Manuscript}

Equilibrium mechanics of monolayered epithelium

Jure Derganc, Saša Svetina, Boštjan Žekš

PII:

S0022-5193(09)00298-7

DOI: doi:10.1016/j.jtbi.2009.06.021

Reference: YJTBI 5611

To appear in:

Journal of Theoretical Biology

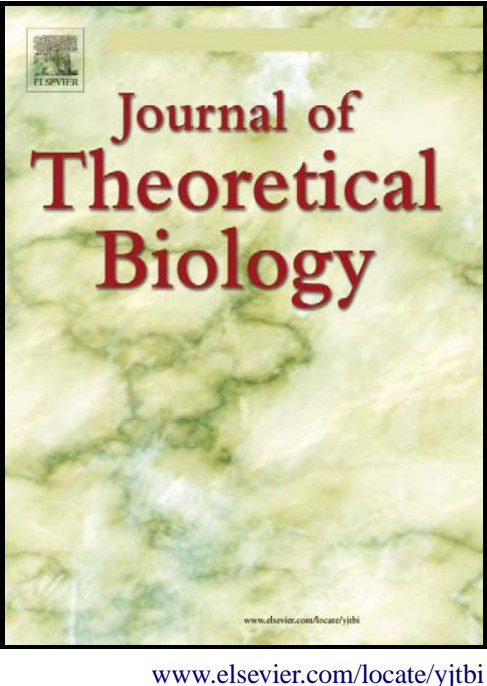

Received date: 11 July 2008

Revised date: $\quad 20$ May 2009

Accepted date: 24 June 2009

Cite this article as: Jure Derganc, Saša Svetina and Boštjan Žekš, Equilibrium mechanics of monolayered epithelium, Journal of Theoretical Biology, doi:10.1016/j.jtbi.2009.06.021

This is a PDF file of an unedited manuscript that has been accepted for publication. As a service to our customers we are providing this early version of the manuscript. The manuscript will undergo copyediting, typesetting, and review of the resulting galley proof before it is published in its final citable form. Please note that during the production process errors may be discovered which could affect the content, and all legal disclaimers that apply to the journal pertain. 


\title{
Equilibrium mechanics of monolayered epithelium
}

\author{
Jure Derganc $^{a, *}$, Saša Svetina ${ }^{a, b}$, and Boštjan Žekšs $\check{c}^{c, a, b}$
}

${ }^{a}$ Institute of Biophysics, Faculty of Medicine, University of Ljubljana, Lipiceva 2, SI-1000 Ljubljana, Slovenia

${ }^{b}$ Jožef Stefan Institute, Jamova cesta 39, SI-1000 Ljubljana, Slovenia

${ }^{c}$ University of Nova Gorica, Vipavska 13, SI-5000 Nova Gorica, Slovenia

May 20, 2009

\footnotetext{
${ }^{*}$ Corresponding author. Tel.: (386) 1543 7615, Fax: (386) 14315127
} 


\begin{abstract}
In order to fully understand the epithelial mechanics it is essential to integrate different levels of epithelial organization. In this work, we propose a theoretical approach for connecting the macroscopic mechanical properties of a monolayered epithelium to the mechanical properties at the cellular level. The analysis is based on the established mechanical models - at the macroscopic scale the epithelium is described within the mechanics of thin layers, while the cellular level is modeled in terms of the cellular surface (cortical) tension and the intercellular adhesion. The macroscopic elastic energy of the epithelium is linked to the energy of an average epithelial cell. The epithelial equilibrium state is determined by energy minimization and the macroscopic elastic moduli are calculated from deformations around the equilibrium. The results indicate that the epithelial equilibrium state is defined by the ratio between the adhesion strength and the cellular surface tension. The lower and the upper bounds for this ratio are estimated. If the ratio is small, the epithelium is cuboidal, if it is large, the epithelium becomes columnar. Importantly, it is found that the cellular cortical tension and the intercellular adhesion alone can not produce the flattened squamous epithelium. Any difference in the surface tension between the apical and basal cellular sides bends the epithelium towards the side with the larger surface tension. Interestingly, the analysis shows that the epithelial area expansivity modulus and the shear modulus depend only on the cellular surface tension and not on the intercellular adhesion. The results are presented in a general analytical form, and are thus applicable to a variety of monolayered epithelia, without relying on the specifics of numerical finite-element methods. In addition, by using the standard theoretical tools for multi-laminar systems, the results can be applied to epithelia consisting of layers with different mechanical properties.

Keywords: cellular mechanics; cellular surface tension; adhesion; bending; cuboidal epithelium; columnar epithelium; squamous epithelium
\end{abstract}




\section{Introduction}

Monolayered epithelium is one of the primary tissues in multicellular animals. Its role begins early in the morphogenesis of a developing embryo and continues as one of the vital structural and functional tissues in mature organisms. Mechanical properties of monolayered epithelium, and particularly the mechanics of epithelial bending, have been long recognized to play an important role in a variety of situations (Ettensohn, 1985). In embryogenesis, for example, epithelial mechanics is closely involved in the primary invagination of the sea urchin blastula (Davidson et al., 1995), while later its importance can be, for instance, recognized during constriction of asthmatic airways (Wiggs et al., 1997).

There are at least three levels of epithelial mechanics. At the macroscopic level, many of the epithelial functions depend directly on how hard it is to stretch or bend the epithelial sheet as a whole. At this level, the monolayered epithelium can be regarded as homogeneous tissue with defined macroscopic elastic moduli. At a lower level, the epithelial mechanics is related to the mechanical properties of individual epithelial cells (Fristrom, 1988), and finally, at the molecular level, the mechanical properties can be attributed to a complex machinery of cytoskeletal and other cellular proteins (Schöck and Perrimon, 2002). At last but not least, the mechanics of multicellular tissues is closely related to a variety of genetic aspects (Hutson and Ma, 2008).

Many complementary models have been proposed to deal with the various levels of epithelial mechanics. For example, at the macroscopic level, the epithelial mechanics has been addressed with numerical finite-element methods (Odell et al., 1981; Davidson et al., 1995; Wiggs et al., 1997), analytical approaches (Murray and Oster, 1984), or even a combination of the two (Brodland et al., 2006). At the cellular level, there are two general approaches (Hoffman et al., 2006): on the one hand, the cellular mechanics can be defined on the basis of the cellular bulk elasticity (Ingber, 2003), while on the other, many of the epithelial phenomena can be described in terms of the cellular surface mechanics (Lecuit and Lenne, 2007). In the later case the epithelial stability is provided by the balance between the cell-cell adhesion and the contractility of the cellular cortex (Janmey and Discher, 2004; Farhadifar et al., 2007; Montell, 2008; Rauzi et al., 2008). At the microscopic level, the intercellular adhesion is related to various adhesion molecules (Steinberg, 
2007), while the cellular cortical tension is related to the submembrane acto-myosin network (Evans and Kukan, 1984). Still, the cellular level has not been effectively connected to the macroscopic scale, and quantitative relations linking the cellular and the macroscopic properties are not well established.

In this work we propose an analytical approach for connecting the macroscopic elasticity of a monolayered epithelium to the properties at the cellular level. The aim of the analysis is to link the established mechanical models from both scales. The mechanical properties at the cellular level are described by the cellular surface mechanics, i.e., by the balance between the intercellular adhesion and cellular contractility. Macroscopically the epithelium is described by the standard approach of the continuum mechanics of thin layers (i.e., membrane mechanics). In contrast to 2-D modeling of epithelial mechanics (Brodland et al., 2006; Farhadifar et al., 2007; Rauzi et al., 2008), the framework of mechanics of thin layers allows for a consistent description of epithelial bending, which is clearly important in epithelial invagination and folding. Explicit quantitative relations that connect the epithelial macroscopic equilibrium and its elastic moduli to the cellular cortical tension and the intercellular adhesion are derived.

The analytical approach has at least two advantages. First, it provides analytical results, which can be applied to a variety of epithelial systems without employing the numerical finite-element methods. Second, in the cases where the epithelial cellular layer is adjacent to other mechanically important layers (e.g., the extracellular matrix), the findings of the analysis presented can be incorporated into the standard theoretical techniques for multi-lamellar systems. For example, the analysis presented can be applied to the studies of the sea urchin blastula invagination, where the cellular layer and the extracellular matrix act jointly as a bending bilayer (Lane et al., 1993; Božič et al., 2006).

The work is organized as follows. In the first step, the four independent macroscopic deformational modes of the epithelium are defined and related to the average cellular shape. In the next step, the cellular mechanical energy is defined on the basis of cellular cortical tension and the intercellular adhesion. Finally, the equilibrium state of epithelium is identified as the state which minimizes the elastic energy, and the macroscopic elastic moduli, i.e., the area extension, 
the in-plane shear and bending moduli, are calculated by analyzing the deformations around the equilibrium (Kozlov and Winterhalter, 1991).

\section{Model}

\subsection{Macroscopic mechanical energy}

On the macroscopic scale, the epithelium will be considered as laterally homogeneous and isotropic. Because the lateral dimensions of a unicellular epithelial layer are larger than its thickness, the epithelium can be treated within the standard approach of membrane mechanics (note that the lateral dimensions of individual cells may in fact be smaller than the epithelial thickness). Accordingly, the macroscopic epithelial deformations will be described in terms of four independent deformational modes, two of which are related to epithelial lateral deformations and two are related to epithelial bending.

The lateral deformational modes are the surface extension, which describes isotropic extension of the epithelial layer surface area, and shear, which describes the in-plane stretching at a constant surface area. Following the notation introduced by Evans and Skalak (1980), the isotropic extension will be described by the variable $\alpha$, which denotes the fractional change in the surface area relative to the equilibrium, $\alpha=\left(A / A_{0}-1\right)$. Similarly, the shear is described by the variable $\beta$, which denotes the change in the in-plane aspect ratio of a deformed surface element when the surface area remains constant, i.e., the aspect ratio of an initially square surface element under shear is $1+\beta$.

The bending modes can be defined in different ways. The standard way in the field of membrane mechanics follows the notion of Helfrich (1973) and defines the surface density of the bending

energy as $\frac{d W_{b}}{d A}=\frac{1}{2} k_{c}\left(C_{1}+C_{2}-C_{0}\right)^{2}+\overline{k_{c}} C_{1} C_{2}$, where $C_{1}$ and $C_{2}$ are the principal membrane curvatures, $k_{c}$ and $\overline{k_{c}}$ are the local and the Gaussian bending constants, respectively, and $C_{0}$ is the spontaneous curvature. This notion is particularly useful for describing closed homogeneous membranes, e.g., lipid vesicles, where the integral of the Gaussian term is a constant and depends only on the membrane topology. The bending energy of nonclosed surfaces, however, can be more conveniently described in terms of the mean curvature $H=\left(C_{1}+C_{2}\right) / 2$ and the deviatoric 
curvature $D=\left(C_{1}-C_{2}\right)$ (Fischer, 1993), $\frac{d W_{b}}{d A}=\frac{1}{2} k_{H}\left(2 H-H_{0}\right)^{2}+\frac{1}{2} k_{D} D^{2}$, where $H_{0}$ is the equilibrium value of the epithelial mean curvature. The constants are related through:

$$
k_{c}=k_{H}+k_{D}, \quad \overline{k_{c}}=-2 k_{D} \text { and } \quad C_{0}=\frac{H_{0} k_{H}}{k_{H}+k_{D}}
$$

For small deformations around equilibrium the macroscopic elastic energy density of a monolayered epithelium can be thus written as:

$$
\frac{d W_{\text {layer }}}{d A_{0}}=\frac{1}{2} K \alpha^{2}+\frac{1}{2} \mu \beta^{2}+\frac{1}{2} k_{H}\left(2 H-H_{0}\right)^{2}+\frac{1}{2} k_{D} D^{2}
$$

where $A_{0}$ is the epithelial equilibrium surface area, $K$ is the area expansivity modulus, and $\mu$ is the in-plane shear modulus. In isotropic layers, the energy lacks the linear deviatoric term as it is invariant to coordinate permutation $\left(C_{1} \rightarrow C_{2}\right.$ and $\left.C_{2} \rightarrow C_{1}\right)$. Similarly, the equilibrium value of the shear variable $\beta$ is zero as well.

The two lateral deformations are mutually independent by definition and the same is true for the two bending deformations. The independence of the lateral and bending deformations (i.e., their orthogonality) can be assured by an appropriately defined neutral plane of the epithelial layer, which is the plane whose surface area does not change under pure bending from equilibrium. All four deformational variables $\alpha, \beta, H$ and $D$ are defined with respect to the neutral plane, and the epithelial surface area $A$ is measured in the neutral plane. With a proper definition of the neutral plane the mixed derivatives of the layer mechanical energy $W_{\text {layer }}$ vanish: $\frac{\partial^{2} W_{\text {layer }}}{\partial \alpha \partial H}=0$, $\frac{\partial^{2} W_{\text {layer }}}{\partial \alpha \partial D}=0, \frac{\partial^{2} W_{\text {layer }}}{\partial \beta \partial H}=0$, and $\frac{\partial^{2} W_{\text {layer }}}{\partial \beta \partial D}=0$, and as a result the energy lacks the mixed terms (Kozlov and Winterhalter, 1991). The neutral plane does not necessarily coincide with the middle plane of the epithelial layer.

\subsection{Cellular geometry and deformations}

In order to connect the macroscopic epithelial elasticity to the cellular level, we will first discuss how an average epithelial cell deforms under macroscopic epithelial deformations. Generally, the cells forming a monolayered epithelium are laterally tightly packed and have prismatic shapes with polygonal bases on their apical/basal sides. Although the hexagonal cellular symmetry is often predominant, the long-range order is broken by variations in the cellular sizes, the number of sides 
and their orientations. In a bent epithelium, the cellular geometries can be even more complex. However, taking into account the macroscopic isotropy and homogeneity of the epithelium, the geometric differences among individual cells are clearly averaged out at the macroscopic scale. Accordingly, it can be assumed that the macroscopic properties of the layer can be associated with an average cellular geometry, which will be described here by a model cell. In this way, the average cellular mechanical energy will be approximated by the mechanical energy of the model cell.

In a flat epithelium, a natural choice for the shape of the model cell would be a uniform hexagonal prism, which would also provide the needed macroscopic lateral isotropy (two dimensional hexagonal lattices are laterally isotropic). However, as there is no uniform way of describing the deformations of a hexagonal prism in a bent epithelium, in this work the model cell will have a simpler cylindrical geometry (Fig. 1A). In this way, the deformations of the model cell will be uniformly defined even in a bent epithelium. Although the cylindrical model cell has inherently a smaller area-to-volume ratio than real epithelial cells with polygonal faces, it can effectively describe an average cellular geometry. Indeed, the use of cylindrical geometry for describing an average epithelial cell in a flat epithelium has been already confirmed by a numerical finite-element analysis (Brodland et al., 2006).

The relations between the macroscopic epithelial deformations and the deformations of the cylindrical model cell can be described in a straightforward manner. As the volume of epithelial cells remains constant during epithelial deformation, it is assumed that the volume of the model cell is constant as well. Under area extension of the epithelial layer the surface areas of the model cell base sides increase and the model cell height decreases (Fig. 1B). A shear deformation stretches the base sides of the model cell into ellipses (Fig. 1C). Bending of the layer stretches the apical and basal sides in the directions of the two principal curvatures while the lateral sides of the model cell are parallel to the curvature radius. When both principal curvatures of the epithelium are equal, the model cell takes the shape of a truncated cone (Fig. 1D), and when the two principal curvatures of the epithelium differ, the apical and basal sides are elliptical with the axes parallel to the principal curvatures (Fig. 1E). In general, all transversal cross-sections of the model cell are elliptical, too (Fig. 2), and the position of the neutral plane is shifted by $\epsilon$ from the layer 
midplane.

It turns out that the geometry of the model cell with a constant volume can be defined by four independent parameters (see Appendix A). Thus, the deformations of the model cell from equilibrium can be uniquely expressed in terms of the four deformational variables $\alpha, \beta, H$ and $D$.

\subsection{Cellular mechanical energy}

After establishing the connection between the cellular and epithelial deformations, the macroscopic epithelial elastic energy can be straightforwardly related to the cellular mechanics. Here, the cellular level will be described in terms of the cellular surface mechanics and the intercellular adhesion energy (Evans and Kukan, 1984; Lecuit and Lenne, 2007). As the epithelial cells have three functionally distinct surfaces (the apical, the basal and the lateral side), each of these surfaces can in general have a different surface energy. Consequently, three independent parameters are needed to describe the overall cellular mechanical energy. Here, the choice of the parameters will follow the notion of the liquid drop model (Evans and Kukan, 1984), where the whole cell surface is under a constant surface (cortical) tension $\sigma$. Accordingly, the mechanical energy of an epithelial cell is written as

$$
W=\sigma\left(A_{\mathrm{A}}+A_{\mathrm{B}}+A_{\mathrm{L}}\right)+\eta\left(A_{\mathrm{B}}-A_{\mathrm{A}}\right)-\frac{1}{2} \gamma A_{\mathrm{L}}
$$

where $A_{\mathrm{A}}, A_{\mathrm{B}}, A_{\mathrm{L}}$ are the surface areas of the apical, basal and lateral sides of the cell, $\sigma$ is the cellular cortical tension, and $\gamma$ is the intercellular adhesion constant. The parameter $\eta$ describes the possible asymmetry between the surface energies of the apical and basal sides.

The macroscopic mechanical energy of the layer (Eq. 2) is equal to the sum of the cellular mechanical energies (Eq. 3) of all cells. Because the model cell represents an average cell in the layer, the macroscopic equilibrium of the epithelium can be thus calculated as the minimum of the mechanical energy of the model cell. Moreover, as the macroscopic deformational modes have been linked to the cellular deformations (the explicit relations between the macroscopic deformational variables $(\alpha, \beta, H$ and $D)$ and the surface areas of the model cell $\left(A_{\mathrm{A}}, A_{\mathrm{B}}, A_{\mathrm{L}}\right)$ are presented in the Appendix A), the macroscopic elastic moduli can be calculated from the changes of the cellular mechanical energy around the equilibrium. 
In order to reduce the number of independent parameters in the model, all quantities will be scaled with respect to the cylindrical model cell with the minimal surface area at a given volume. This "unit" model cell represents a cuboidal epithelial cell, which has the same height and width. The unit length is thus the base radius of the "unit" model cell $R_{c}$ (its height and width are twice $R_{c}$ ), the unit surface area is $\pi R_{c}^{2}$, and the unit volume is $2 \pi R_{c}^{3}$. The unit energy is chosen to be $\sigma \pi R_{c}^{2}$. The dimensionless variables will be written in lowercase: the dimensionless surface area is $a=A /\left(\pi R_{c}^{2}\right)$, the dimensionless layer thickness $l=L / R_{c}$, and the dimensionless curvatures $h=H R_{c}$ and $d=D R_{c}$. The lateral deformational variables $\alpha$ and $\beta$ are dimensionless by definition. The mechanical energy assigned to the epithelial model cell (Eq. 3) in the dimensionless form is thus:

$$
w=\left(a_{\mathrm{A}}+a_{\mathrm{B}}+a_{\mathrm{L}}\right)-\frac{1}{2} \bar{\gamma} a_{\mathrm{L}}+\bar{\eta}\left(a_{\mathrm{B}}-a_{\mathrm{A}}\right)
$$

where $a_{\mathrm{A}}=A_{\mathrm{A}} /\left(\pi R_{c}^{2}\right), a_{\mathrm{B}}=A_{\mathrm{B}} /\left(\pi R_{c}^{2}\right), a_{\mathrm{L}}=A_{\mathrm{L}} /\left(\pi R_{c}^{2}\right), \bar{\gamma}=\gamma / \sigma$ and $\bar{\eta}=\eta / \sigma$.

The dimensionless notation reveals that the system has only two independent parameters: the ratio between the adhesion constant and the surface tension $\bar{\gamma}$, and the parameter describing asymmetry between the apical and basal side $\bar{\eta}$.

\section{Results}

\subsection{Equilibrium state of free epithelia}

In absence of external forces, the equilibrium state of the epithelium is defined by the minimum of its total mechanical energy. According to the assumptions of the model, the minimum of the total energy coincides with the minimum of the energy of the model cell. Clearly, due to symmetry considerations, the equilibrium value of the deformational variables $d$ in $\beta$ is zero. The equilibrium values of the mean layer curvature $h_{0}$ and the surface area of the neutral plane $a_{0}$ are calculated

from $\frac{\partial w}{\partial h}=0$ and $\frac{\partial w}{\partial \alpha}=0$. The position of the neutral plane $\epsilon$ is defined by $\frac{\partial^{2} w}{\partial \alpha \partial h}=0$, while all other mixed derivatives vanish automatically. In the limit of small curvatures and small asymmetry between the basal and apical side $(\bar{\eta} \ll 1)$, the equilibrium surface area of the neutral plane $a_{0}$, 
the equilibrium layer thickness $l_{0}$ and the mean spontaneous curvature $h_{0}$ are:

$$
\begin{aligned}
a_{0} & =\left(\frac{2-\bar{\gamma}}{2}\right)^{2 / 3} \\
l_{0} & =\frac{2}{a_{0}} \\
h_{0} & =\bar{\eta} \frac{3 a_{0}}{3 a_{0}{ }^{3}+1}
\end{aligned}
$$

while the relative shift of the neutral plane from the layer's midplane is

$$
\epsilon=\bar{\eta} \frac{3 a_{0}^{3}+4}{18 a_{0}^{3}+6}
$$

It is informative to express also the cellular height-to-width ratio:

$$
\text { height-to-width ratio }=\frac{l_{0}}{2 \sqrt{a_{0}}}=a_{0}^{-3 / 2}=\frac{1}{1-\frac{1}{2} \bar{\gamma}}
$$

Fig. 3 shows the equilibrium values of the geometric variables as functions of the relative adhesion strenght $\bar{\gamma}$ at five different values of $\bar{\eta}$. As expected, when there is no difference between the basal and apical sides $(\bar{\eta}=0)$, the epithelium spontaneous mean curvature $h_{0}$ is zero and the neutral plane is in the middle of the layer, $\epsilon=0$. At vanishing adhesion strenght $(\bar{\gamma}=0)$, the cells take the "cuboidal" shape, i.e., the shape with the minimal total cellular surface area $\left(a_{0}=1\right.$, $l_{0}=2$ and height-to-width ratio $=1$, Fig. $3, \mathrm{~A}$ and $\left.\mathrm{B}\right)$. As $\bar{\gamma}$ increases, the cells become thinner and the epithelia thicker. Since the height-to-width ratio is larger than 1 at all values of $\bar{\gamma}$, the model can not describe the flattened squamous epithelia. Assuming that the height-to-width ratio of columnar epithelial cells is on the order of 10 , Fig. 3B reveals that the ratio $\gamma / \sigma$ in these systems is not larger than $\bar{\gamma} \approx 1.8$. The model predicts that at $\bar{\gamma}=2$ the epithelium becomes infinitely thick with $a_{0}=0$.

When the apical and basal sides have different surface energies $(\bar{\eta} \neq 0)$ the epithelium bends towards the side with a larger surface tension - the layer spontaneous curvature $h_{0}$ is proportional to $\bar{\eta}$ (Fig. $3 \mathrm{C}$ ). Note, however, that in the limit $\bar{\eta} \ll 1, a_{0}$ and $l_{0}$ do not depend on $\bar{\eta}$. The deviation of the neutral plane from the middle is at most a few percent (Fig. 3 D).

At small adhesion strengths, the mechanical energy per cell in the layer may become larger than the energy of a corresponding free non-adhered spherical epithelial cell (at a given volume, a spherical cell has a smaller total surface area than a cell in the layer). Thus, if the adhesion 
strength is bellow a certain threshold value, it is not energetically favorable for the cells to form the layer at all. A simple calculation (not shown) yields that the corresponding threshold value of the adhesion strength is $\bar{\gamma} \approx 0.37$.

\section{$3.2 \quad$ Elastic moduli}

The elastic moduli are the second derivatives of the mechanical energy (Eq. 3) with respect to the deformational variables around the equilibrium: $K=\frac{1}{A_{0}} \frac{\partial^{2} W}{\partial \alpha^{2}}, \mu=\frac{1}{A_{0}} \frac{\partial^{2} W}{\partial \beta^{2}}, k_{H}=\frac{1}{4 A_{0}} \frac{\partial^{2} W}{\partial H^{2}}, k_{D}=$ $\frac{1}{A_{0}} \frac{\partial^{2} W}{\partial D^{2}}$. In contrast to the equilibrium geometrical parameters, which were suitably presented in the dimensionless form, the calculated elastic moduli are more informative when presented in the dimensional form:

$$
\begin{aligned}
K & =3 \sigma \\
\mu & =\frac{3}{2} \sigma \\
k_{H} & =\frac{1}{4}\left(a_{0}^{3}+\frac{1}{3}\right) \sigma L_{0}^{2} \\
k_{D} & =\frac{1}{8}\left(a_{0}^{3}-\frac{1}{3}\right) \sigma L_{0}^{2} .
\end{aligned}
$$

where the dimensionless equilibrium surface area $a_{0}$ is defined by Eq. 5 , and $L_{0}$ is the dimensional epithelial thickness in equilibrium. The local bending modulus $k_{c}$ and Gaussian bending modulus $\overline{k_{c}}$ can be then calculated from Eqs. 1 :

$$
\begin{aligned}
k_{c} & =\frac{1}{8}\left(3 a_{0}^{3}+\frac{1}{3}\right) \sigma L_{0}^{2}, \\
\overline{k_{c}} & =\frac{1}{4}\left(\frac{1}{3}-a_{0}^{3}\right) \sigma L_{0}^{2} .
\end{aligned}
$$

The results show that the lateral elastic moduli $K$ and $\mu$ are proportional to the cellular cortical tension $\sigma$ and that they do not depend on the intercellular adhesion $\gamma$. The dependence of the bending elastic moduli on the relative adhesion constant $\bar{\gamma}$ is presented in Fig. 4. Clearly, the bending constants diverge as $\bar{\gamma}$ increases. This divergence can be regarded as a direct consequence of the divergent layer thickness $L_{0}$ (Fig. 3B and Eqs. 14 and 15). 


\section{Discussion}

This work introduces a novel analytical approach for analyzing the relations between the cellular and the macroscopic mechanical properties of epithelium. While some of the relations between the cellular and macroscopic levels have been qualitatively well understood (Forgacs and Newman, 2005), the analysis presented is an attempt to express these relations in a quantitative manner. The cellular parameters are described via cellular cortical tension $\sigma$ and an effective intercellular adhesion constant $\gamma$, which are phenomenological parameters linked to the activity of the submembrane acto-myosin network and cell adhesion molecules, respectively (Evans and Kukan, 1984; Steinberg, 2007). Since the macroscopic level is described within the standard theory of continuum mechanics, the analysis can serve as a general analytical framework for assessing macroscopic epithelial mechanics from the point of view of cellular processes, without relying on the specific numerical finite-element methods.

The analysis reveals that the main factor in the macroscopic equilibrium of free and laterally homogeneous epithelia is the balance between the adhesion constant $\gamma$ and the cellular cortical tension $\sigma$, and provides the relation between the epithelial thickness and $\gamma / \sigma$. The lower and the upper bounds for $\gamma / \sigma$ are estimated: if the ratio $\gamma / \sigma$ is small, the epithelial cells are cuboidal (i.e., with minimal cellular surface area at a given volume), when this ratio increases, the cells become columnar and the layer thickens in order to increase the intercellular contact surface area (Fig. 3, A and B). The layer has a finite thickness only if the ratio $\gamma / \sigma$ is smaller than 2. Clearly, this divergence is not present in real systems and it can be expected that, in order to increase the contact surface area at very large adhesion strengths, the cells in real systems take shapes other than the one assumed by the model (e.g., even non-planar 3-D cellular aggregates may become energetically favorable). On the other hand, if the adhesion is too weak $(\gamma / \sigma<0.37)$, the energy of individual non-adhered spherical cells becomes smaller than the energy of the cells in the epithelium and the formation of epithelium is not energetically favorable.

Importantly, the model predicts that the cellular height-to-width ratio is larger than 1 at all values of $\gamma / \sigma$ (Fig. 3B). This indicates that the cortical tension and the intercellular adhesion alone can not produce spontaneous cell flattening, where the lateral cellular dimensions become 
larger than the epithelial thickness. The flattened cellular geometries are, for example, observed in squamous epithelia or in the morphogenesis of the Drosophila wing discs (Fristrom, 1988). In these cases, however, the epithelium is not free and the flattening could be a result of a strong adhesion to a substrate on the apical/basal sides. Of note, these findings agree well with the observed dependence of the follicle cell shape on the expression of E-cadherin (Montell, 2008).

The elastic moduli of epithelia are calculated from the deformations around the equilibrium. The calculation shows that the epithelial area expansivity modulus is directly proportional to the cellular cortical tension $\sigma$ (Eq. 10), and that the in-plane shear modulus $\mu$ is one half of the area expansivity modulus (Eq. 11). This relation $(K=2 \mu)$ is typical for two-dimensional isotropic materials (Boal, 2002). Interestingly, $K$ and $\mu$ do not depend on the intercellular adhesion. Again, it can be shown that this result applies to all prismatic cellular geometries (see Appendix B). These important and unexpected results call for a further experimental investigation. The analysis also shows that the layer bending constant is proportional to the area expansivity modulus and the square of the layer thickness, $k_{c} \approx K L_{0}^{2}$, as is often the case in conventional materials. The Gaussian bending constant, however, is negative at small values of $\gamma / \sigma$ and positive at larger $\gamma / \sigma$ (Fig. 4B). Note that the detailed role of the Gaussian bending constant in folding of nonclosed surfaces remains to be analyzed.

The analytical nature of the model presented enables a straightforward analysis of experimental images of the epithelial cross-section. For example, the experiments by Davidson et al. (1999) show that a typical blastula of a developing sea urchin embryo is spherical, with a radius $R_{b} \approx 50 \mu \mathrm{m}$, epithelial thickness (i.e., the cellular height) $L_{0} \approx 6 \mu \mathrm{m}$, and the cellular height-to-width ratio 5:3. Assuming that the blastula is near the equilibrium, the ratio between the adhesion and the cortical tension $\bar{\gamma}$ can be estimated directly from the cellular geometry in the epithelial cross-section, i.e., from the cellular height-to-width (see Eq. 9 and Fig. 3B), $\bar{\gamma}=2\left(1-\frac{1}{\text { h-to-w }}\right) \approx 0.8$. Once the ratio $\bar{\gamma}$ is known, the in-plane cellular surface area can be estimated, $a_{0} \approx 0.7$ (see Eq. 5 and Fig. 3A) and the dimensionless cellular height can be calculated from Eq. $6, l_{0}=2 / a_{0} \approx 2.8$. Then, the unit length can be determined from the actual cellular height, $R_{c}=L_{0} / l_{0} \approx 2.1 \mu \mathrm{m}$. Finally, the dimensionless epithelial mean curvature is $h_{0}=H_{0} R_{c}=2 / R_{b} \times R_{c} \approx 0.1$ (for a spherical blastula, 
the mean curvature is twice the inverse of the epithelial radius of curvature, $H_{0}=2 / R_{b}$ ), which yields $\bar{\eta} \approx 0.05$ (see Eq. 7 and Fig. 3C). As the measured upper limit for the epithelial Young modulus was $700 \mathrm{~Pa}$ (Davidson et al., 1999), the corresponding upper limits for $\sigma$ and $\gamma$ are of the order of magnitude $1 \mathrm{pN} / \mu \mathrm{m}\left(10^{-15} \mathrm{~J} / \mu \mathrm{m}^{2}\right)$. While we are not aware of any direct measurements of the cortical tension in epithelia, the estimated value agrees with the value found in fibroblasts, $\sigma \approx 0.3 \mathrm{pN} / \mu \mathrm{m}$ (Thoumine et al., 1999). Interestingly, the estimated value of $\bar{\gamma} \approx 0.8$ indicates a small Gaussian bending modulus (Fig. 4B).

The cellular level was modeled by the cellular surface mechanics (Beysens et al., 2000; Janmey and Discher, 2004), which completely neglects the cellular bulk elasticity. In the first approximation, the use of the surface mechanics is justified if the cells have flat cellular sides, without any noticeable undulations (i.e., if the cellular surfaces are minimized). Epithelial cells in the sea urchin blastula, for example, indeed show flat sides indicating the presence of the surface tension (Davidson et al., 1999).

The analysis presented focused only on the Hookean elasticity regime, i.e., on the small deformations of a homogeneous epithelium around the equilibrium, where the epithelial cells are in a relaxed state and their behavior does not depend on their more complex features (e.g., viscosity). However, the approach based on average cellular properties can be extended to cover a broader set of epithelial behavior, provided that the epithelium (or a patch of the epithelium in question) can be considered laterally homogeneous at the macroscopic level. For example, the macroscopic lateral anisotropy of epithelia can be introduced by defining more than four macroscopic elastic moduli and more than three microscopic parameters. In order to describe larger deformations on wider time scales, one can still analyze the behavior of the model cell by taking into account the non-Hookean elasticity terms (i.e., higher derivatives of the mechanical energy) and the viscous behavior of the tissue. Specifically, the fluid-like cellular rearrangements on longer time scales can be described by a relaxation parameter, as has been demonstrated in a numerical analysis of large in-plane epithelial deformations (Brodland et al., 2006). Also, it remains to be analyzed if the role of non-uniformly distributed intercellular adhesion complexes in epithelial bending (e.g., adherens junctions) can be described solely by an effective adhesion constant and an appropriate position 
of the layer neutral plane.

The geometry of the cylindrical model cell does not describe the actual shapes of individual epithelial cells and it should be regarded as a first order approximation of the average shape behavior. Yet, there are several indications that this simplification, made in a manner of a mean field approximation, is a reasonable one. For example, the results regarding the in-plane deformations are not artifacts of the cylindrical geometry but are valid for all prismatic model cell geometries (see Appendix B). Also, the use of the cylindrical average cell geometry in a flat epithelium has been already confirmed by a numerical finite-element analysis (Brodland et al., 2006). Moreover, there is good qualitative agreement between the experimentally observed cellular deformations during the primary invagination of the sea urchin blastula (Kimberly and Hardin, 1998) and the predicted model cell geometries. For example, the conical bottle-shaped cells are found in the epithelial regions where both curvatures negative (Fig. 1D), and an elongation of the cellular apical sides in the radial direction is observed at the orifice of the invagination, where the epithelium is bent only in the radial direction.

Finally, it should be noted that the macroscopic mechanical properties of epithelial tissues do not depend only on the layer of epithelial cells, but also on the adjacent material layers possessing markedly different mechanical properties, e.g., the extracellular matrix. For example, one of the proposed mechanisms of epithelial invagination is in fact related to the bilayer bending effect of the cellular layer and the extracellular matrix (Lane et al., 1993). Following the approach that was applied in the analysis presented, these complex epithelial organizations can be readily described by the standard continuum mechanics of multi-laminar systems. Indeed, by taking into account the diverse properties of the cellular layer, the apical lamina and the hyaline layer, a multi-laminar analytical approach has already provided a novel insight into blastula mechanics and yielded general mechanical conditions that have to be fulfilled during the invagination (Božič et al., 2006). Thus, the quantitative analytical approach can bypass the elaborate finite-element modeling of multi-layered systems, used for example in the analysis of the mechanical compression of the blastula wall (Davidson et al., 1995, 1999), and bring an alternative point of view to the interpretations of experimental investigations of epithelial mechanics. 


\section{Acknowledgments}

This work was supported by Slovenian Research Agency Grant P1-0055. 


\section{A Geometry of the model cell}

The geometry of the model cell is described in Fig. 2. In general, the apical and basal sides are elliptical with the principal radii $R_{1}, R_{2 A}$ and $R_{1_{B}}, R_{2 B}$ (indices 1 and 2 represent the directions of the two principal curvatures, and the indices $A, B$ and $N$ represent the apical, basal and neutral plane, respectively). The principal radii of other cross-sections change linearly with the cellular height.

The epithelial principal curvatures and the principal elliptical radii of the model cell base sides are related through (Fig. 2):

$$
\begin{array}{cc}
\tan \alpha_{1}=\frac{R_{1 A}-R_{1 B}}{L} \quad, \quad \tan \alpha_{2}=\frac{R_{2 A}-R_{2 B}}{L} \\
R_{1 N}=\frac{1}{C_{1}} \sin \alpha_{1} \quad, \quad R_{2 N}=\frac{1}{C_{2}} \sin \alpha_{2}
\end{array}
$$

The volume of the model cell can be calculated as

$$
V=\int_{z=0}^{z=L} \pi R_{1}(z) R_{2}(z) d z
$$

where the principal elliptical radii of the model cell cross-section $R_{1}$ and $R_{2}$ are

$$
\begin{aligned}
& R_{1}(z)=R_{1 B}+\frac{z}{L}\left(R_{1 A}-R_{1 B}\right) \\
& R_{2}(z)=R_{2 B}+\frac{z}{L}\left(R_{2 A}-R_{2 B}\right)
\end{aligned}
$$

The apical surface area is $A_{A}=\pi R_{1 A} R_{2 A}$, the basal surface area is $A_{B}=\pi R_{1 B} R_{2 B}$, while the lateral surface area can be calculated as:

$$
A_{L}=\int_{z=0}^{z=L} \int_{\phi=0}^{\phi=2 \pi} d A_{L}
$$

where the lateral surface element $d A_{L}$ can be calculated from

$$
d A_{L}=\sqrt{{\overrightarrow{R_{L \phi}}}^{2}{\overrightarrow{R_{L z}}}^{2}-\left(\overrightarrow{R_{L z}} \overrightarrow{R_{L \phi}}\right)^{2}}
$$

Here, the vector defining the lateral surface is

$$
\overrightarrow{R_{L}}=\left(\begin{array}{c}
R_{1}(z) \cos \phi \\
R_{2}(z) \sin \phi \\
z
\end{array}\right)
$$


The principal radii can be expressed in terms of $A$ and $\beta\left(A=\pi R_{1 N} R_{2 N}\right.$ and $\beta=R_{2 N} / R_{1 N}-$ 1), and the principal curvatures $C_{1}$ and $C_{2}$ can be expressed in terms of $H$ and $D$. As the volume of the model cell is constant, the cellular height can be calculated form Eq. A-3, and therefore the model cell geometry is uniformly defined by four parameters. As a result, in the limit of small curvatures and small shear, the surface areas of the model cell can be expressed as (in the dimensionless form):

$$
\begin{aligned}
& a_{A}=a+(2-4 \epsilon) h+\frac{h^{2}}{a}-\frac{d^{2}}{4 a}, \\
& a_{B}=a-(2-4 \epsilon) h+\frac{h^{2}}{a}-\frac{d^{2}}{4 a}, \\
& a_{L}=\frac{4}{\sqrt{a}}+\frac{8 \epsilon}{a^{3 / 2}} h+\frac{2\left(3 a^{3}-2\right)}{3 a^{5 / 2}} h^{2}+\frac{5+3 a^{3}}{12 a^{5 / 2}} d^{2}+\frac{3}{4 \sqrt{a}} \beta^{2} .
\end{aligned}
$$

The mechanical energy of the model cell is then calculated by combining Eqs. A-9-A-11, and Eq. 4. The relation between the deformational variable $\alpha$ and the layer surface area $a$ is $\alpha=\left(a / a_{0}-1\right)$

\section{B Area extension modulus in a general prismatic geometry}

It can be easily verified that in flat epithelia, the value of the area extension modulus, $K=3 \sigma$ (Eq.10), is the same for all uniform prismatic model cell geometries. In the flat epithelium, the apical and the basal faces of the model cell are equal, $A_{A}=A_{B}=A$, and the cellular lateral surface area equals the perimeter of the base face times the cellular height. The perimeter is proportional to the square root of $A$, and since the cellular volume is constant, the cellular height is inversely proportional to $A$. It follows that $A_{L}=\frac{C}{\sqrt{A}}$, where $C$ is a constant. The cellular energy (Eq. 3) then reads (neglecting the apico-basal asymmetry):

$$
W_{\text {flat }}=\sigma\left(2 A+\frac{C}{\sqrt{A}}\right)-\frac{1}{2} \gamma \frac{C}{\sqrt{A}}
$$

Minimizing the energy with respect to $A$ gives

$$
A_{0}=\frac{1}{4} C^{2 / 3}\left(2-\frac{\gamma}{\sigma}\right)^{2 / 3}
$$

and the calculation of the area extension modulus $\left(K=A_{0} \frac{\partial^{2} W}{\partial A^{2}}\right)$ then leads to Eq.10. 


\section{References}

Beysens, D. A., Forgacs, G., and Glazier, J. A. (2000), Proc. Natl. Acad. Sci. USA 97, 9467

Boal, D. (2002), Mechanics of the Cell, Cambridge, UK: Cambridge University Press

Božič, B., Derganc, J., and Svetina, S. (2006) , Int. J. Dev. Biol. 50, 143

Brodland, G. W., Chen, D. I. L., and Veldhuis, J. H. (2006) , Int. J. Plasticity 22, 965

Davidson, L. A., Koehl, M. A., Keller, R., and Oster, G. F. (1995) , Development 121, 2005

Davidson, L. A., Oster, G. F., Keller, R. E., and Koehl, M. A. (1999) , Dev. Biol. 209, 221

Ettensohn, C. A. (1985), Q. Rev. Biol. 60, 289

Evans, E. and Kukan, B. (1984) , Blood 64, 1028

Evans, E. and Skalak, R. (1980), Mechanics and Thermodynamics of Biomembranes, Boca Raton FL: CRC Press

Farhadifar, R., Röper, J. C., Aigouy, B., Eaton, S., and Jülicher, F. (2007) , Curr. Biol. 17, 2095

Fischer, T. M. (1993) , J. Phys. II France 3, 1795

Forgacs, G. and Newman, S. A. (2005), Biological Physics of the Developing Embryo, Cambridge UK: Cambridge University Press

Fristrom, D. (1988), Tissue \& Cell 20, 645

Helfrich, W. (1973), Z. Naturforsch. 28c, 693

Hoffman, B. D., Massiera, G., Citters, K. M. V., and Crocker, J. C. (2006) , Proc. Natl. Acad. Sci. USA 103, 10259

Hutson, M. S. and Ma, X. (2008), Phys. Biol. 5, 15001

Ingber, D. E. (2003) , J. Cell Sci. 116, 1157

Janmey, P. A. and Discher, D. E. (2004), Nature 431, 635 
Kimberly, E. L. and Hardin, J. (1998) , Dev. Biol. 204, 235

Kozlov, M. M. and Winterhalter, M. (1991) , J. Phys. II France 1, 1077

Lane, M. C., Koehl, M. A. R., Wilt, F., and Keller, R. (1993), Development 117, 1049

Lecuit, T. and Lenne, P. F. (2007), Nat. Rev. Mol. Cell Biol. 8, 633

Montell, D. J. (2008) , Science 322, 1502

Murray, J. D. and Oster, G. F. (1984), Math. Med. Biol. 1, 51

Odell, G. M., Oster, G., Alberch, P., and Burnside, B. (1981), Dev. Biol. 85, 446

Rauzi, M., Verant, P., Lecuit, T., and Lenne, P. F. (2008), Nat. Cell Biol. 10, 1401

Schöck, F. and Perrimon, N. (2002), Annu. Rev. Cell Dev. Biol. 18, 463

Steinberg, M. S. (2007), Curr. Opin. Genet. Dev. 17, 281

Thoumine, O. O., Cardoso, O., and Meister, J. J. (1999) , Eur. Biophys. J. 28, 222

Wiggs, B. R., Hrousis, C. A., Drazen, J. M., and Kamm, R. D. (1997), J. Appl. Physiol. 83, 1814 


\section{Figure Legends}

\section{Figure 1.}

Schematic representation of the model cell geometry when the epithelium is subject to different deformational modes. The model cell shape can be regarded as an average shape of the cells in the epithelial layer. The top and the bottom faces of the model cell represent the cellular apical and basal sides, respectively, and the side face of the model cell represents the lateral cellular sides. The cellular height is the same as the epithelial thickness, and the cellular width is the cellular diameter in the epithelial plane. Macroscopic deformations of the layer cause deformations of the apical, basal and lateral sides of the model cell, and do not alter the cellular volume. (A) A cylindrical model cell in a flat layer; (B) cell flattening due to the layer area extension; (C) in-plane shear: the base faces are deformed into matching ellipses; (D) isotropic bending of the layer $(H \neq 0, D=0)$; and $(\mathrm{E})$ the saddle (deviatoric) deformation $(H=0, D \neq 0)$ : the base faces are deformed into ellipses with perpendicular major axes.

\section{Figure 2.}

Longitudinal and transversal cross-sections of the model cell in the case when the epithelium is under saddle deformation (Fig. 1E). (A) The longitudinal cross-sections along the directions of the two principal curvatures $C_{1}$ and $C_{2}$. The thickness of epithelium equals the height of the model cell $L$; (B) The cross-sections in the apical, basal and neutral planes. The indices 1 and 2 represent the directions of the two principal curvatures, and the indices $A, B$ and $N$ represent the apical, basal and neutral plane, respectively. Note that the neutral plane does not necessarily coincide with the layer midplane.

\section{Figure 3.}

Equilibrium geometric parameters of the epithelium as a function of the ratio between the adhesion constant $\gamma$ and the cellular cortical tension $\sigma$. (A) Relative surface area per cell in the epithelial neutral plane, $a_{0}=A_{0} / \pi R_{c}^{2}$ (Eq. 5); (B) cellular height-to-width ratio (Eq. 9); (C) relative equilibrium mean epithelial curvature, $h_{0}=H_{0} R_{c}$ (Eq. 7); (D) the relative shift of the neutral 
plane from the layer midplane (Eq. 8), as defined in Fig. 2. The epithelial curvature and the relative shift of the neutral plane are shown at five values of the parameter $\bar{\eta}$, which describes the apicobasal asymmetry $(\bar{\eta}=0, \pm 0,05$ and $\pm 0,1)$. Note that the equilibrium surface area and thickness do not depend on $\bar{\eta}$. The values are scaled with respect to $R_{c}$, which is the radius of the base face of the "unit" model cell, which represents a cuboidal epithelial cell, i.e. the cell with the minimal surface area at a given volume (see text).

\section{Figure 4.}

The epithelial bending elastic moduli as a function of the ratio between the adhesion constant $\gamma$ and the cellular cortical tension $\sigma$. (A) the local bending constant $k_{c}$; (B) the Gaussian bending constant $\overline{k_{c}}$. The values are scaled with respect to $R_{c}$, which is the radius of the base face of the "unit" model cell, which represents a cuboidal epithelial cell, i.e. the cell with the minimal surface area at a given volume (see text). 
Figure 1

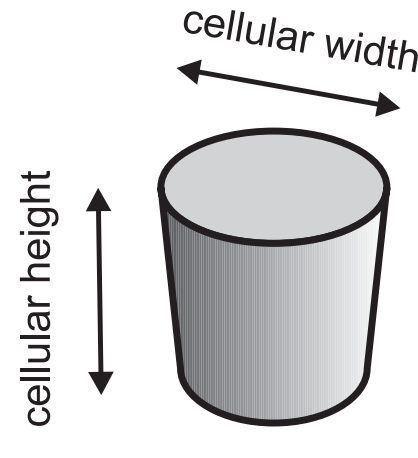

A

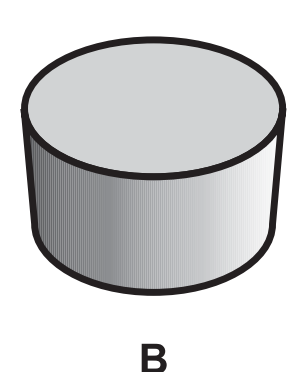

B
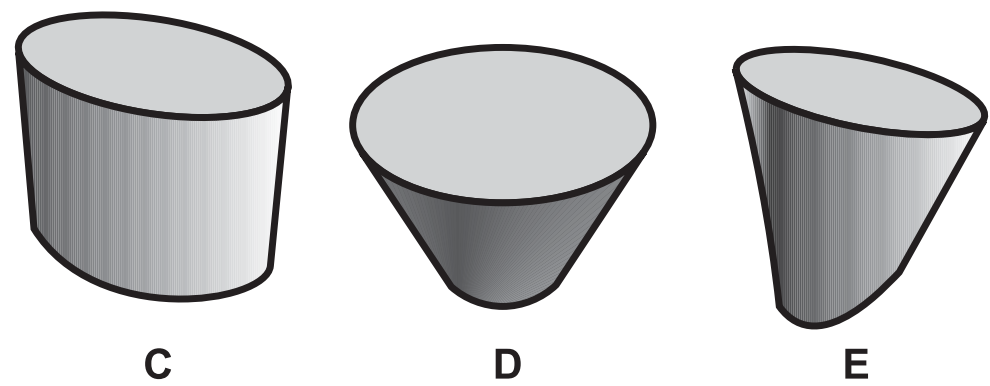
A

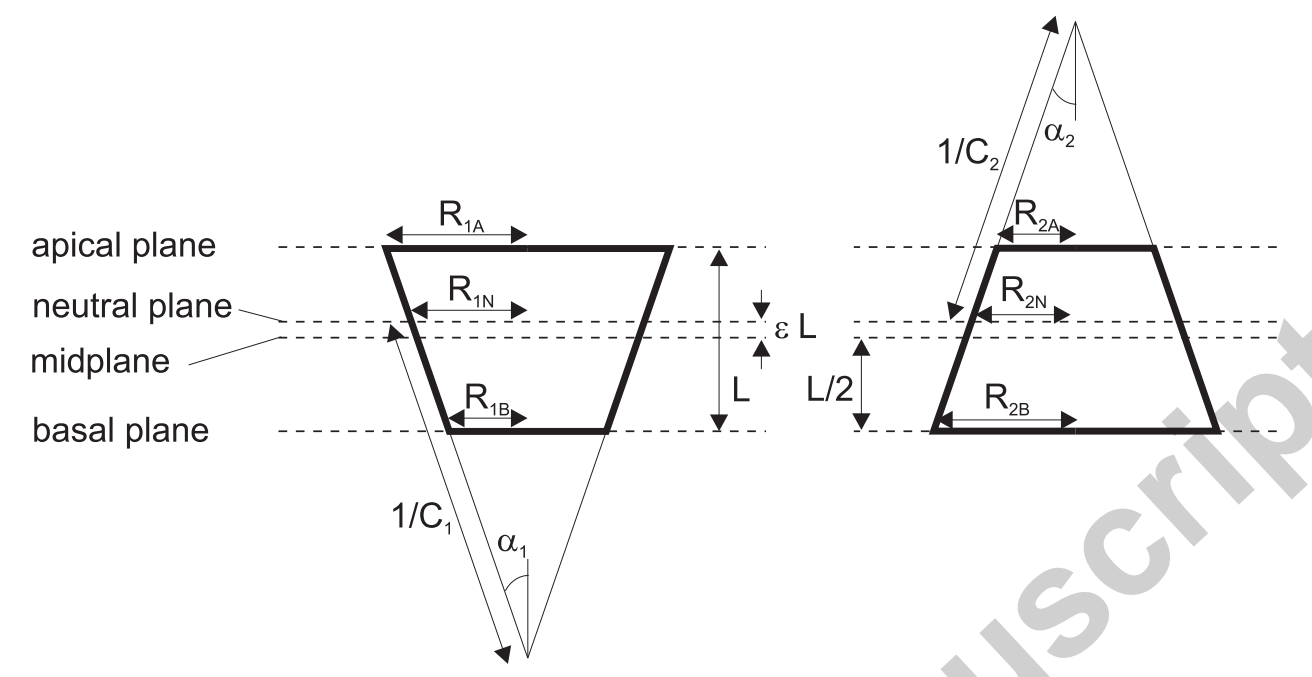

B
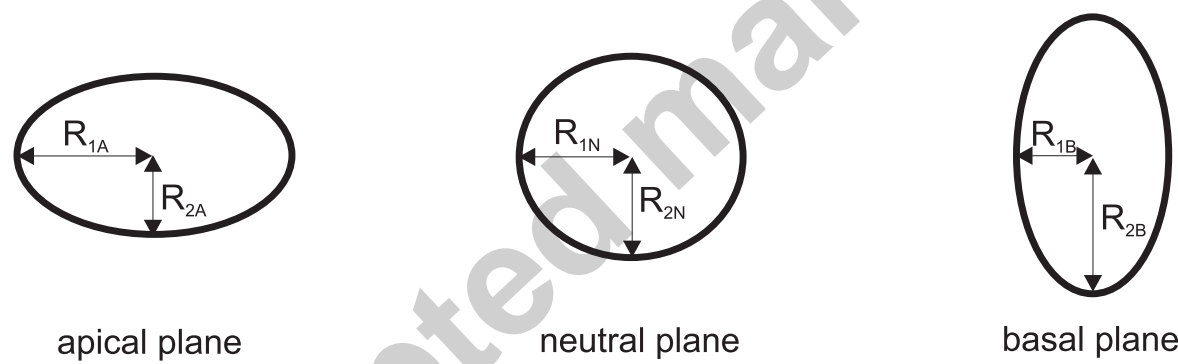

apical plane

neutral plane

basal plane 
Figure 3
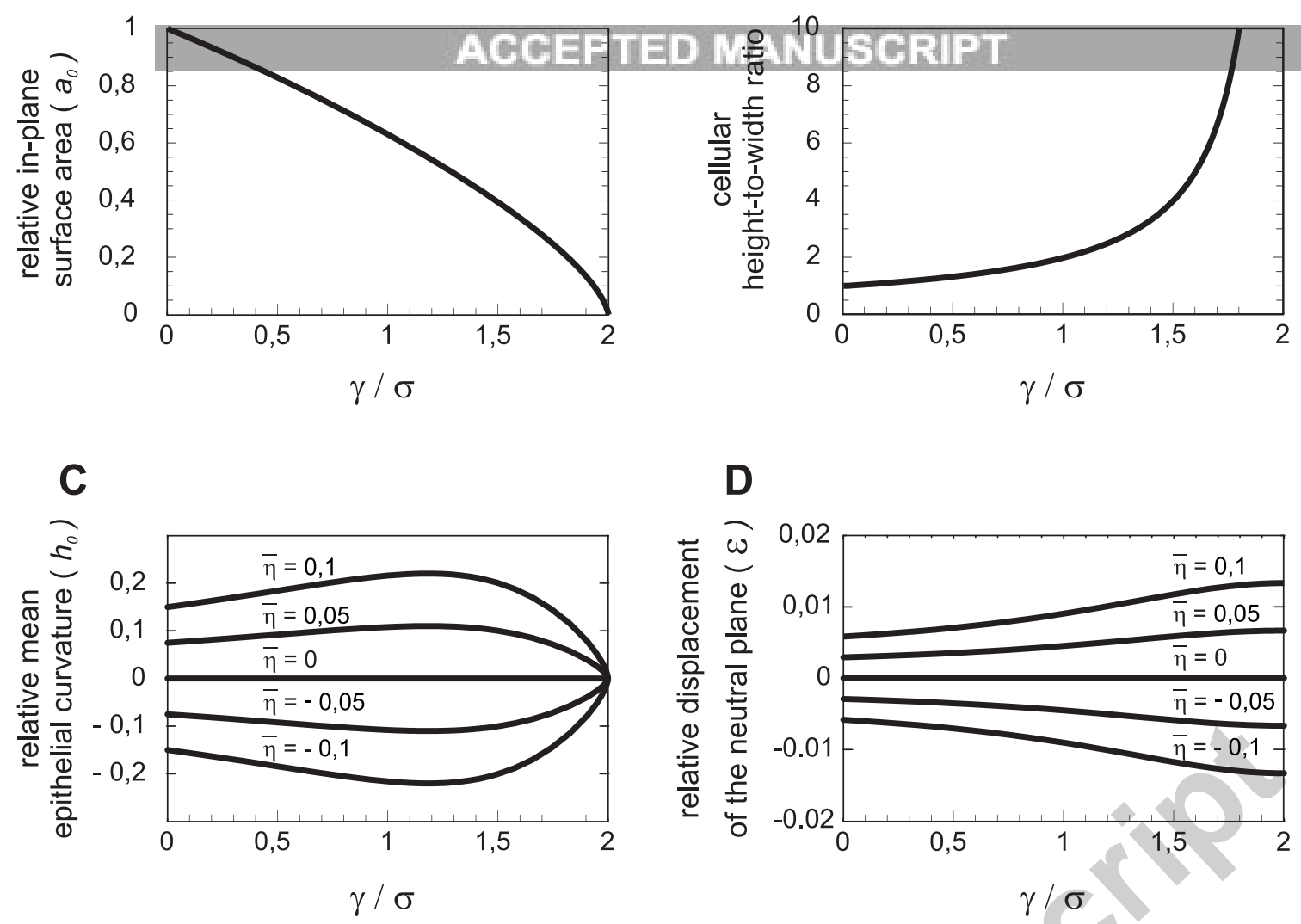
A
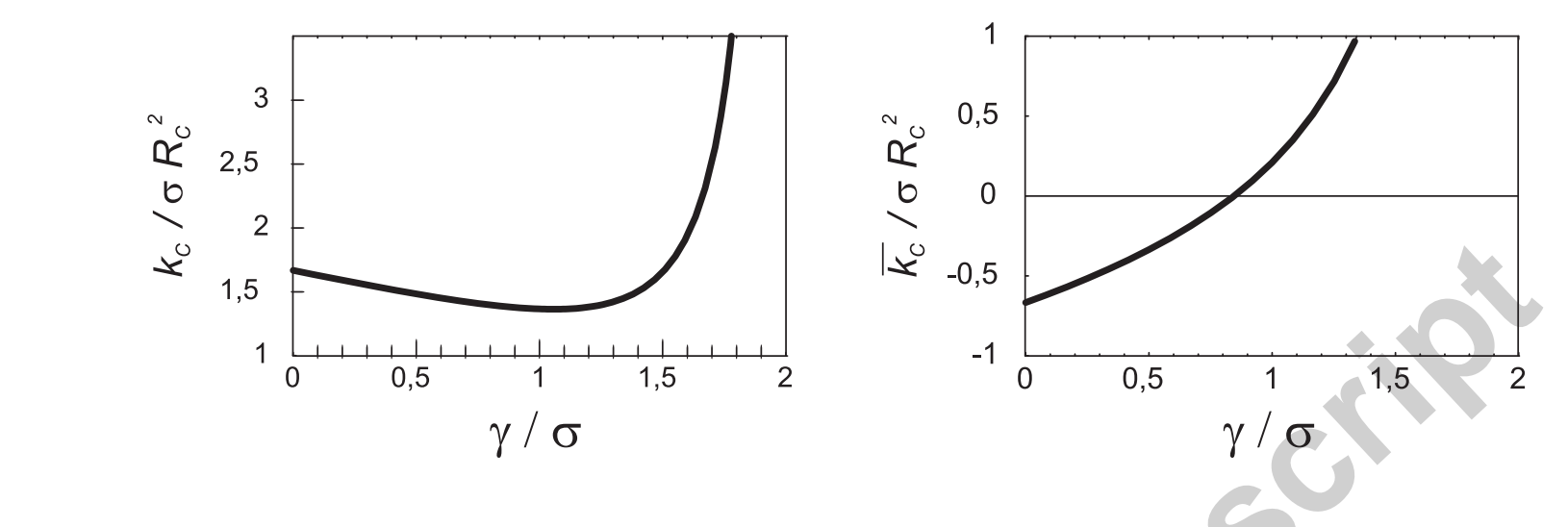

Figure 4
B

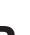

.
A

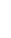

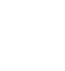

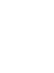

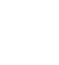

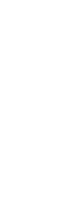

(
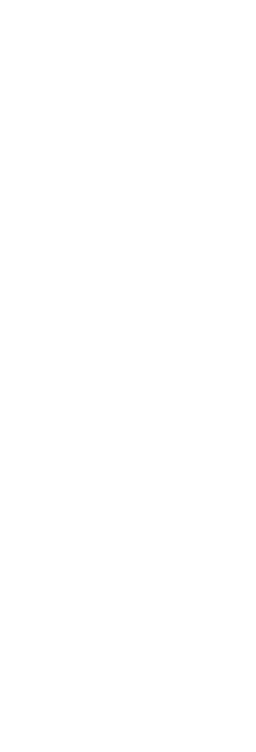

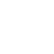

